\section{ÜBRIGENS}

... ist die Hypothyreose häufigste Komplikation nach Krebs im Kindesalter. Nach einem Morbus Hodgkin liegt der Anteil bei $27,5 \%$ (Hyperthyreose: $3,1 \%$ )

Monatsschr Kinderheilkd 2015; 163; 120-125

... unterscheidet sich der IQ bei Kindern mit angebrochener Hypothyreose nicht von dem gesunder Geschwister, wenn die L-T4-Therapie mit mindestens $10 \mathrm{~g} / \mathrm{kg}$ nach der Geburt begonnen wird.

Best Pract \& Res Clin Endocrinol \& Metabol 2015; 29; 399-413

... wird in der TRUST-Studie über fünf Jahre geprüft, wie sich die Gesundheit bei Älteren mit subklinischer Hypothyreose maßgeblich verbessern lässt; Infos auf:

www.trustthyroidtrial. com.

Punkte sammeln mit der Sommerakademie!

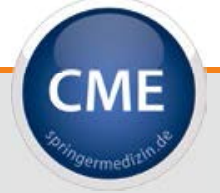

Zum Thema "Schilddrüse" finden Sie unter www.springermedizin.de/sommerakademie den Kurs:

„Differenzierte Schilddrüsenkarzinome”

Mit e.Med teilnehmen

Mit e.Med haben Sie nicht nur Zugriff auf die aktuelle Sommerakademie, Sie können das komplette Angebot von Springer Medizin nutzen - zusätzlich erhalten Sie eine gedruckte Fachzeitschrift Ihrer Wahl. Mit rund 1600 Fortbildungskursen, davon über 500 CME-zertifiziert, ist die e.Akademie Deutschlands erste Adresse für Online-CME-Fortbildung.

Nutzen Sie die Sommerzeit, um reichlich CME-Punkte zu sammeln!

30 Tage kostenlos

Unser Kundenservice steht Ihnen wochentags (10-17 Uhr) kostenlos zur Verfügung: Tel. 08007780777 kundenservice@springermedizin.de

Jeder Dritte ist gefährdet

\title{
Kombitherapie verkleinert Struma
}

Noch hat nicht jeder in Deutschland eine optimale Jodversorgung. Jodpräparate helfen hier weiter. Bei Strumen lässt sich eine Volumenreduktion gut durch eine Kombitherapie erreichen.

Mit einem Anteil von 90\% ist die euthyreote Struma weiterhin die häufigste Schilddrüsenerkrankung. Deutschland sei zwar den Ergebnissen des Gesundheitssurveys für Erwachsene (DEGS) des Robert KochInstituts zufolge derzeit kein Jodmangelgebiet, so der Arbeitskreis Jodmangel. Diese Aussage gelte jedoch nur für den Bevölkerungsdurchschnitt. Das Jodmonitoring habe gezeigt, dass bei etwa einem Drittel der Menschen in Deutschland die Jodversorgung nicht ausreicht.

Die durch das fehlende Jod vergrößerte Schilddrüse lässt sich durch eine Behandlung mit Jodid (etwa Jodidratiopharm ${ }^{\circ}$ ) bei einem Teil der Patienten

\section{Prospektive Studie}

\section{Jodexzess für Schilddrüse ohne Folgen}

Kann zu viel Jod eine Funktionsstörung der Schilddrüse triggern? Eine neue Studie bestätigt das nicht.

Die Befürchtung, durch eine einmalige übermäßige Jodexposition eine Hypooder Hyperthyreose auszulösen, ist einer prospektiven Studie zufolge offenbar unbegründet. In der Studie mit insgesamt 102 schilddrüsengesunden erwachsenen Patienten, die sich einer Untersuchung mit der Computertomographie unterzogen, kam es nur bei $2 \%$ danach zu einer subklinischen Hyperthyreose. Der Schilddrüsenfunktionstest wurden vier bis acht Wochen später gemacht. Wie die Endokrino-

logen von der Universität von Auckland in Neuseeland berichten, entwickelte sich nur bei zwei Patienten erstmals eine subklinische Hyperthyreose mit normalen Werten für freies T3 und freies T4.

\section{Zusammenhang mit Bildgebung}

Die neuseeländischen Endokrinologen erinnern daran, dass die exzessive Jodaufnahme im Zusammenhang mit der Bildgebung durch Kontrastmittel 200.000fach höher lag als die Empfehlungen für den Tagesbedarf $(180 \mu \mathrm{g}$ bei Erwachsenen). um etwa $20 \%$ verkleinern. Weil sich das Hormon und das Spurenelement ergänzten, werde häufig auch die Kombinationstherapie Jodid plus L-Thyroxin vorgenommen. Zur Verfügung stehen Präparate wie Thyronajod ${ }^{\circ}$ und Eferox ${ }^{\bullet}$ Jod $^{\circ}$.

\section{Kombitherapie punktet im Vergleich} Dass mit der Kombinationstherapie im Vergleich zu den Komponenten plus Placebo mehr zu erreichen ist, ging bereits aus der LISA-Studie hervor. Darin wurde die Wirksamkeit einer Therapie mit $75 \mu \mathrm{g}$ L-Thyroxin, der Kombination aus $75 \mu \mathrm{g}$ T4 und $150 \mu$ g Jodid und einer Monotherapie mit der gleichen Dosis von Jodid und Placebo geprüft. Im Vergleich zur Placebogruppe war die Volumenreduktion bei den Patienten, die die Kombinationstherapie erhielten, signifikant und am stärksten ausgeprägt (17,3\%).

(ple)

J Clin Endocrinol Metab 2011; 96: 2786-2795 